\title{
A dynamic systems approach to personality: The Personality Dynamics
}

\section{(PersDyn) model}

\begin{abstract}
In the present paper, we offer an integrative approach to personality that combines withinperson and between-person differences. By drawing on the principles of dynamic systems theory, we present Personality Dynamics model - a novel framework that captures people's typical pattern of changes in personality states using three model parameters: baseline personality, reflecting the stable set point around which one's states fluctuate, personality variability, or the extent to which one's personality states fluctuate across time and situations, and personality attractor force, pertaining to the swiftness with which deviations of one's baseline are pulled back to the baseline. We argue that the dynamic approach to personality represented in the PersDyn model has the potential to integrate different perspectives on individual differences. We also demonstrate that the dynamic approach to personality offers a consensual paradigm of personality with the potential to advance our understanding and knowledge of individual differences, by detailing the factors and processes included in the model, as well as links to existing theories and applications in various research lines.
\end{abstract}

\section{Personality as a dynamic system}

The dynamic character of personality has been acknowledged in research for a very long time already. Even in early writings on the topic (e.g., Allport, 1937), it was emphasized that personality is a dynamic, psychological organization that coordinates our experiences and actions. Yet, despite this awareness, personality research has focused almost exclusively on the study of stable, between-person differences in broad dispositions that are invariant over time and unaffected by situational influences. Such an approach, although undoubtfully useful, strips personality from its dynamic character. In the present paper, we argue that the 
introduction of this dynamic element in personality theory requires an integrative approach to personality: An approach that incorporates both traits and states, thereby reconciling both the stable and dynamic aspect of personality.

In the present paper, we offer such an integrative approach to personality by drawing on the principles of dynamic systems theory. A dynamic system is, by definition, a set of interconnected elements that evolve over time due to dynamic interactions between the system's elements. In terms of personality, a dynamic system of personality thus represents a set of personality elements that interact with each other, with such interactions resulting in the behavioural, affective and cognitive manifestations of traits. In a dynamic system of personality, the elements of the system can be understood as broad trait dimensions (e.g., the Big Five personality dimensions), but also as more narrow elements (e.g., the Big Five trait facets underlying those broad dimensions, including more specific cognitions, emotions, and behaviors). Besides arguing that studying personality through the lens of dynamic systems theory is useful because it allows incorporating different levels of analysis (i.e., trait level vs. state level), regulatory mechanisms, as well as dynamic interactions between the elements of the system, and changes over time, a second important contribution of the present paper is the introduction of a personality model based on the dynamical systems approach that is both comprehensive and empirically testable (i.e., the Personality Dynamics (PersDyn) model). By doing this, we hope to demonstrate the potential of dynamic systems theory in offering a unifying, empirically applicable framework for personality science.

In what follows, we first discuss how the PersDyn model captures personality dynamics, reviewing the major building blocks of the model. Next, we describe what distinguishes the dynamic systems approach from the traditional approach to individual differences (see Table 1 for a systematic comparision of both approaches), and we discuss how the PersDyn model accommodates for existing personality theories, ranging from 
traditional, structural models (e.g., Five Factor Model, McCrae \& Costa, 1999; HEXACO, Lee \& Ashton, 2004) to more dynamic personality theories (e.g., network models, Cervone, 2005; Cramer et al., 2012; Read, Monroe, Brownstein, Yang, Chopra, \& Miller, 2010). Finally, we present applications of the model in different areas of psychological research, and we discuss challenges and future research directions.

\section{The Personality Dynamics model}

Although it is challenging to conceptualize personality using the principles of dynamic systems theory, we will introduce a model that builds on its assumptions in a relatively straightforward and empirically testable way: the Personality Dynamics model (PersDyn; Sosnowska, Hofmans, \& De Fruyt, 2016). The PersDyn model describes people's typical pattern of changes in one's personality space. In the model, one's trajectory of personality states is captured by means of three model parameters: baseline, variability, and attractor strength. Baseline represents the average level of behaviour, affect and cognitions [baseline personality, reflecting one's personality trait level] and it serves as the point of reference in the system. Variability, in turn, pertains to the extent to which the individual varies around this point of reference and captures the extent to which the individual exhibits different behaviours, affects and cognitions over time [personality variability, reflecting variability in one's personality states]. People with low variability mostly act the same, while others might be highly variable in their personality states. Attractor strength, finally, bridges stability and variability because it represents the swiftness with which the individual returns to his/her homebase once he/she has deviated from it [personality attractor strength, reflecting personality regulation]. When deviations from the baseline are regulated swiftly, one has a strong attractor, while with a weak attractor return to the baseline goes slowly. 
The PersDyn model builds on the DynAffect model of Kuppens, Oravecz, and Tuerlinckx (2010), a model that describes people's typical pattern of changes on the two dimensions of core affect: valence and arousal. In the PersDyn model, the parameters do not necessarily capture movements in two-dimensional core affect space, but rather changes on either a single personality dimension (see Figure 1) or a set of personality dimensions. That is, although the PersDyn can be used to capture changes on a single personality dimension, in theory the model also allows looking into complex interactions between the elements of different personality traits, using for example the Big 5 or HEXACO personality dimensions.

In what follows, we will briefly describe each building block of the PersDyn model and discuss how these building blocks represent meaningful elements of one's personality system.

Personality baseline. The baseline in the PersDyn model represents the central point around which the individual's behaviours, thoughts and emotions fluctuate (see Figure 1, representing one's personality states across time on a single personality dimension). This baseline can be derived from a series of momentary personality states, and represents how the person behaves, thinks and feels on average (i.e., across time and across situations). Figure 1 shows such individual differences in personality baseline in four hypothetical trajectories, with trajectories 1.1 and 1.2 being examples of trajectories from individuals with a high baseline level, while trajectories 1.3 and 1.4 result from people with a low baseline level. Because baseline personality pertains to how one generally (or on average) behaves, feels and thinks, the baseline represents the state toward which one's behaviours, feelings and cognitions tend to converge ${ }^{1}$. Therefore, it plays an important role in self-regulation of the system, serving as the reference point that maintains the system's stability (Vallacher \& Nowak, 2005). That the mean level of one's momentary trait expressions over time offers a

\footnotetext{
${ }^{1}$ The baseline may be also subject to change over time (normatively or idiosyncratically).
} 
meaningful way to describe personality has been recognized by several leading personality researchers and personality models. For example, in the density distribution approach (Fleeson, 2001), the central point of the distribution has been shown to vary between individuals and has been demonstrated to be relatively stable across time. Moreover, Fleeson (2001) demonstrated that individual differences in the mean level of momentary trait expressions corresponded well with trait differences as measured by traditional trait questionnaires. In other theories, baseline personality has been conceived of as an attractor a set of stable states towards which the system converges over time (i.e., Shoda, LeeTiernan and Mischel, 2002). Similarly, the baseline has been proposed in the concept of a psychological profile as well (Furr, 2009), where it represents the general trait level aggregated across situations.

Personality variability. The second component of the model, personality variability, represents the extent to which the person's behaviours, feelings and cognitions fluctuate over time, capturing the state component of personality. In Figure 1, trajectories 1.1 and 1.2 result from people characterized by high personality variability, while trajectories 1.3 and 1.4 are indicative of low personality variability. Deviations from one's baseline (i.e., variability) happen because of the impact of situational forces and internal motivations, which pull one's behaviours, feelings and cognitions away from the baseline. For example, a person may be a dispositional introvert (low extraversion baseline), but due to situational forces (e.g., being at a party or having a selection interview) or internal motives (e.g., a momentary urge to relate to others), (s)he may momentarily behave in an extraverted way, thereby veering away from his/her baseline. Variability comes, therefore, from interactions with different situations, environments and internal forces (i.e., personality dimensions impacting upon the expression of other personality dimensions). 
Interestingly, research on personality variability has revealed that individual differences in personality variability are stable over time, implying that they can be used to characterize and compare people (i.e., Mõttus, Epskamp, \& Francis, 2017; Orom \& Cervone, 2009). As a result, variability is often referred to as 'consistency in inconsistency' (Roberts, 2009), as there are stable between-person differences in the extent to which people change their behaviours, feelings and cognitions. Recently, personality variability has also been referred to as personality strength (Dalal, Meyer, Bradshaw, Gree, Kelly, \& Zhu, 2015), with people who fluctuate less being less susceptible to external and internal forces, implying that they have a "stronger personality". Finally, studies on personality variability have shown that personality variability shows low to moderate correlations across traits (Dalal et al., 2015; Fleeson, 2001). This finding suggests that personality variability cannot be reduced to either a response bias and that it is not a direct effect of other traits, such as sensation seeking. In fact, Fleeson (2001) found personality variability to reflect individual differences in the reactivity to situational cues, with people showing more personality variability on a specific personality dimension when the person is more sensitive to situational cues relevant to that dimension.

Personality attractor strength. The final building block of the model, attractor strength, pertains to the regulatory forces that pull the fluctuations back to one's personality baseline. That is, personality attractor strength represents how fast one returns to his/her baseline after they deviated from it. In Figure 1, trajectories 1.1 and 1.3 are trajectories showcasing high attractor strength because in these trajectories one quickly returns to the baseline level once (s)he has deviated from it, while trajectories 1.2. and 1.4 are examples of low attractor strength because of the slow return to the baseline. Attractor strength plays an important role in the personality system as it bridges stability (i.e., returning to one's baseline) and change (i.e., moving away from one's baseline). In fact, thanks to the existence of such a self-regulatory component, the system is able to balance stability of the baseline 
with constant perturbations of the system (or variation around this baseline). As with personality baseline and variability, attractor strength is most likely to be trait-specific, meaning that an individual can have a strong attractor for one trait, but a weak attractor for another trait; e.g., returning fast to a highly extraverted baseline, but returning slowly to a highly conscientious baseline.

Although the notion that people actively regulate their own behaviour, thinking and feelings is not new, attractor strength received substantially less attention in personality research relative to baseline personality and personality variability. However, despite the lack of familiarity with the concept of attractor strength in the personality literature, related concepts have been introduced, such as the cybernetic mechanisms underlying behavioural selfregulation in DeYoung's (2015) Cybernetic Big Five Theory theory, or the basin of attraction in Vallacher, Nowak, Froehlich, and Rockloff's (2002) dynamical system model, with this basin of attraction representing how strong each state that deviates from one's baseline will be pulled back to the baseline.

\section{Processes in the PersDyn model}

Conceptualising personality as a dynamic system implies that, as opposed to the traditional focus on stable predispositions, the focus is on the complex behavioural, cognitive and affective patterns resulting from interactions between different elements of the system (i.e., self-organization of the system; Kelso, 1995). In the PersDyn model these behavioural patterns can be observed from a series of momentary trait expressions - or a series of personality states. Based on the information encompassed by this series of personality states (including the temporal dynamics of the states), higher-level between-person information is inferred (see Figure 2 for differences between such a bottom-up approach and the traditional top-down approach to personality). Thus, between-person differences in personality baseline, personality variability and attractor strength are reflected in and inferred from the 
behavioural, cognitive and affective patterns that emerge over time, with patterns of changes on the within-person level creating meaningful between-person differences (Revelle \& Condon, 2015).

Looking at personality as a process that unfolds over time is possible through the inclusion of a temporal dimension in the model. The inclusion of this temporal dimension is what probably most clearly distinghuishes the traditional and the dynamic approach to personality - by tracking changes in one's behaviours, cognitions and feelings over time, it becomes possible to capture personality processes. In the PersDyn model, attractor strength plays an important role in capturing those personality processes because this is the one PersDyn element that is sensitive to temporal information, linking stability in the system to variability. It is important to note that the inclusion of a temporal dimension allows for the examination and comparison of changes in personality over different time periods. For example, interactions and changes that occur over a short period of time may differ from interactions and changes that occur over longer periods of time. Therefore, it is important to consider how the timeframe might affect the observed phenomena.

Finally, one should note that, although according to dynamic systems thinking personality is a process that unfolds over time in a meaningful way, the changes in behaviour, thinking and feeling can be highly non-linear. This nonlinearity results from the unique interactions between different elements of the model, including interactions between specific parameters of a particular trait (e.g., neuroticism attractor strength interacting with neuroticism baseline) or between the system and its environment (e.g., situational factors interacting with one's personality baseline). Any manifestation of personality traits thus typically results from the interplay of multiple determinants.

\section{Self-regulation in the PersDyn model}


Self-regulation in the PersDyn model is possible because of the existence of an attractor - a fixed point towards which the individual's thoughts, feelings, and predispositions converge. In the PersDyn model, this role is played by the personality baseline, representing the idea that, although personality states are inherently dynamic due to internal and external causes, they tend to move towards the baseline over time. Hence, the baseline serves as a point of reference for self-regulation by representing stability and resistance to change in the personality system. It is important to note that those attractors may or may not correspond to desired end-states. For example, whereas a high neuroticism baseline is not what is typically considered desirable, research has shown that people high on neuroticism tend to return to this baseline, in part because high trait neuroticism predisposes them to experience more stressful (Bolger \& Schilling, 1991) and negative events (Magnus, Diener, Fujita, \& Payot, 1993). In summary, conceptualizing personality traits as attractors encapsulates our innate tendency to develop and maintain stability, while it also allows for behavioural flexibility resulting from interactions with one's environment.

An attractor can develop in two ways: (1) systematic synchronisation of individual's states over time (the automatic-reflexive level), and (2) self-organization of thoughts, feelings and behaviours in order to achieve a goal or adhere to a self-concept (the controlled-reflective level). The first mechanism is based on the idea that attractors are created by repetitive experiences of a particular state or set of states (e.g., highly extroverted behaviours), after which the state becomes 'engraved' in the individual's personality system (Nowak, Vallacher, \& Zochowski, 2005). In other words, cognitive and emotional schemes triggered by the individual's behaviours, feelings and cognitions are reinforced each time the individual acts, feels or thinks that way, which, in turn, makes it more likely that future behaviours, feelings and cognitions fall within a similar range. Moreover, emotional and cognitive reactions to certain events create bi-directional feedback loops, where emotions 
trigger a cognitive reaction that is congruent with them, while cognitive processes trigger emotions that are coherent with the content of one's cognitive appraisals (Lewis, 2005). Such loops have self-amplifying effects, meaning that the behavioural reactions may grow stronger over time. Therefore, an attractor for a particular trait or set of trait-relevant behaviours emerges over time following repeated instances of similar behaviours, feelings and congitions, with self-regulation being an automatic process of following previously reinforced patterns.

The second mechanism responsible for shaping the attractor and self-regulatory forces is based on our capacity for self-reflection and regulation of thoughts, emotions and actions (Baumeister \& Twenge, 2003). As opposed to the automatic process of self-regulation, it puts self-awareness and self-concept in personality on the foreground and it underscores the active role an individual can play in gradually shaping their own personality. For example, people may consciously and actively choose certain behaviours in order to achieve a certain goal (e.g., an introvert socialising during a work event to broaden his/her professional network) or to adhere to a self-concept (e.g., actively reinforcing cognitive and emotional schemes that are coherent with one's self-concept). Through the conscious and active selection of certain and avoidance of other situations, certain behavioural, affective and cognitive patterns can get reinforced (Bolger \& Schilling, 1991), which can strengthen self-regulatory forces on the controlled-reflective level.

\section{Links between the PersDyn model and other personality theories}

The PersDyn model connects to several important lines of research in personality, related to both the structure and processes of individual differences. First and foremost, the model can be applied to any individual difference variable that changes over time (i.e., that is manifested on the state level), which means it accommodates traditional trait models such as Five Factor Model (McRae \& Costa, 1999) or HEXACO (Lee \& Ashton, 2004). Although 
the PersDyn model goes beyond traditional mean-level differences, the personality baseline represents the average behavioural, cognitive and affective tendency, in about the same way as trait-based approaches do. Therefore, the model can easily be reconciled with the more traditional theories on personality traits.

Furthermore, the PersDyn model also accommodates for other descriptive frameworks on personality, such as Fleeson's (2001) density distribution approach and it's recent outgrowth Whole Trait Theory (Fleeson \& Jayawickreme, 2015). The density distribution approach and Whole Trait Theory are build on the idea that people differ from each other not only regarding their average trait level, but also in the extent to which their personality states vary (Debusscher et al., 2016; Jones, Brown, Serfass, Sherman, 2017), the most frequent states, the minimum, starting level of the states, and the state where the individual is pushed to under extreme pressure (Fleeson, \& Gallagher, 2009; Jones et al., 2017). Indeed, studies drawing on these frameworks have shown that personality is not limited to individual differences in one's average behaviours, feelings and cognitions, but that other characteristics of one's density distribution often provide additional information about their behavioural tendencies. The most important difference with the PersDyn model is the fact that the density distribution approach and Whole Trait Theory do not look at the temporal order of the states, while this is a crucial element of the PersDyn model that is reflected in the concept of attractor strength.

Moving on to more dynamic theories on personality, the PersDyn model builds on the aforementioned research on attractors (e.g., Nowak, Vallacher, Tesser, \& Zochowski, 2000; Nowak, Vallacher, \& Zochowski, 2005). The dynamical perspective represented by these attractors captures stability and change in personality processes by acknowledging the role of self-regulation over time. Indeed, the conceptualisation of personality in the PersDyn model and the idea of attractor dynamics is very similar, as they both look at how behavioural, 
affective and cognitive tendencies emerge over time. Furthermore, the dynamic systems approach to personality also shows parallels with network models of personality (Cervone, 2005; Cramer et al., 2012; Read et al., 2010) in the sense that both rely on a bottom-up approach (i.e., they start from micro-level observations, which are then aggregated to the person-level). A network perspective is based on the idea that personality emerges from the connectivity structure of different elements of personality (Cramer et al, 2012). In line with the dynamic systems approach, the individual's behaviours, feelings and cognitions are not due to the existence of fixed predispositions, but they rather emerge from interactions and feedback loops between affective, cognitive and behavioural components.

Finally, there are also conceptual similarities between the PersDyn model and the conditional approach to studying person-situation interactions, such as proposed in the cognitive-affective processing system (CAPS) model of personality (Mischel \& Shoda, 1995, 1998, 1999). According to the CAPS model, the psychological elements of situations trigger emotional and cognitive responses (cognitive affective units) that, in turn, activate certain behaviours. Because those relationships differ between individuals, each individual can be described through stable sets of 'if... then' contingencies. Although the PersDyn model does not allow to directly capture those 'if... then' contingencies, both approaches are similar on a conceptual level in the sense that they both conceptualize personality as a set of behavioural predispositions with certain conditions and boundaries, rather than fixed predispositions that evoke similar behaviours across time and situations.

\section{Applications of the PersDyn model}

In many subfields of psychological science, dynamic systems theory has been used as a general framework to study specific phenomena, such as decision-making in sports (Johnson, 2006), teacher-student dynamics in educational psychology (O'Connor, 2010), learning-teaching trajectories (Steenbeek \& Van Geert, 2013), the development and learning 
of motor coordination in sports psychology (Corbetta \& Vereijken, 1999) and bilateral leg movements in young children in the field of motor development (Thelen, Skala, \& Kelso, 1987). We are convinced that the dynamic approach to personality as represented in the PersDyn model can be applied even beyond those lines of psychological research, including clinical settings (e.g., research on personality pathology, bipolar disorder), social psychology (e.g., affect dynamics and social relationships), and work and organizational psychology (e.g., studies on performance, burnout and engagement). In what follows, we will briefly explain how dynamic systems thinking in general and the PersDyn model in particular can be used to further our knowledge in the aforementioned areas of research.

\section{Clinical psychology}

In clinical settings, the dynamic systems approach to personality might be particularly useful when studying personality disorders (PD) because of its ability to capture stability and change in the behavioural, cognitive and affective manifestations of those disorders (Wright, Hopwood, \& Simms, 2015). Results from various studies (Sadler, Woody, McDonald, Lizdek \& Little, 2015; Johnson \& Nowak, 2002) already demonstrated that, through capturing how symptoms arise and change as a result of dynamic processes and interactions between person and situations, the study of dynamic behavioural patterns is crucial to our understanding of the manifestations of personality pathology. For example, attractor-based research on the dynamic behavioural patterns characterizing bipolar depression has shown that individual differences in the instability of these patterns was related to important outcomes, such as the severity of depression and suicidality (Johnson \& Nowak, 2002).

The PersDyn model has the potential to advance the field of clinical psychology in several ways. First, it can be used to examine patterns of stability and change in personality pathology. In doing so, the model offers an important advantage over previous research because it maps those changes along a temporal dimension. That way, the PersDyn model 
allows capturing not only the behavioural symptoms of the disorder, but also the extent to which the individual is able to regulate them. Questions that can be addressed include: how fast do people recover from severe episodes of PDs? What (person- and environment-related) factors impact the swiftness of self-regulation in PDs? Is self-regulation linked with severity and symptoms of PDs? The PersDyn model can also be used to study stability and variability of personality pathology using different time windows. This is important, as changes that occur on the daily level may differ from changes that occur over longer periods of time. An approach that takes into account the timeframe of those changes certainly has the potential to provide a more comprehensive picture of PDs.

\section{Social psychology}

The dynamic systems approach can also be useful in social psychology, where most phenomena are believed to emerge from interactions between people and are therefore hard to understand using traditional, static approaches. Both individual and group actions depend on a large number of interlinked elements (e.g., individual differences, situational factors) and the interactions between those elements are inherently dynamic, which naturally fits well with a dynamic systems approach. So far, studies in the domain of social psychology have used the dynamic systems approach to study phenomena such as social interdependence (Hegselman, 1994), social change and transitions (Nowak, Urbaniak, Zienkowski, 1994), and social identity (Vugt \& Hart, 2004).

The PersDyn model can be easily adapted to examine social psychological phenomena. One way to do so is applying the model to the behavioural, cognitive and affective manifestations of personality traits in different social contexts. For example, one might study how patterns of behavioural, affective and cognitive manifestations of extraversion differ across social settings (i.e., work, family, social group). Moreover, the 
model can be also used to examine phenomena other than personality traits, such as attitudes, motivations and emotions.

\section{Work and organizational psychology}

The dynamic approach to individual differences offers particularly promising insights in the work domain, where personality is often used by both scientists and practitioners to predict work-related attitudes and behaviours. Research on within-person fluctuations in personality states has already been applied in various work-related studies, including studies on job performance (Debusscher, Hofmans, \& De Fruyt, 2014, 2016a, 2016b, 2016c, 2017; Huang \& Ryan, 2011), learning transfer (Huang \& Bramble, 2016), mood and job satisfaction (Judge \& Ilies, 2002), and work motivation (Judge, Simon, Hurst, \& Kelly, 2014). The concepts of within-person stability and change as captured by the PersDyn model have the potential to move research on the dynamics of work attitudes and behaviours forward, which in the end hopefully improves our selection procedures (see the review on personality dynamics in selection, by Sosnowska, Hofmans, \& Lievens, in press). Up until today, the PersDyn model has been applied in studies on the relationship between personality dynamics and work vigour (Sosnowska, Hofmans, \& De Fruyt, 2017), burnout and exhaustion (Sosnowska, Hofmans, \& De Fruyt, 2015) and performance (Sosnowska \& Hofmans, 2017). As opposed to the traditional trait approach, which emphasises the role of individual personality predispositions in work behaviours (e.g., high conscientiousness linked with high performance), this approach acknowledges the role of changes in personality states and allows studying how those changes affect work behaviours. In general, these studiesalthough few in number-show that within-person fluctuations in personality (represented by personality variability and attractor strength as captured by the PersDyn model) are instrumental in the prediction of work behaviours such as burnout and work performance.

\section{Challenges facing the field and future research agenda}


The dynamic systems approach to personality, and particularly its operationalization in terms of the PersDyn model, offers a novel way to address some of the challenges that personality field is facing now (e.g., the lack of unifying frameworks and theories). However, due to its novel and complex character, the PersDyn model comes with a number of methodological issues. Therefore, in this part of the paper, we discuss challenges that personality research is facing now, as well as ways in which our approach offers a response to those issues.

\section{Theoretical issues}

One of the main challenges that personality research is facing, is the lack of unifying frameworks and theories. For decades, the field of personality psychology has been divided, focusing on different debates such as the trait vs. state debate or the person vs. situation one. Although most researchers are no longer fixated on such debates and although it is by now widely acknowledged that embracing one side does not imply rejecting the other, the links between existing theories are often still missing or only vaguely specified. To overcome this challenge, it is necessary to link personality theories and models across different domains and approaches. In addition to tackling this issue on a conceptual level (which is what is done in the current paper), researchers might also use techniques such as computational modelling. The idea of computational modeling is to use computer simulations to examine how the representation of the system behaves over time, including how the interactions between the elements evolve, thereby allowing to manipulate parameters and conditions that are rather challenging to manipulate in real life. That way, computational modelling allows detecting the compatibility of different theoretical approaches, potentially resolving long-standing theoretical issues. As such, it is a promising line of research within the personality domain.

\section{Methodological issues}

Secondly, personality dynamics are difficult to capture using traditional research 
methods (e.g., cross-sectional research) and analysis techniques (e.g., regression analysis), which do not fit well with an approach that focuses on dynamic interactions and temporal changes. For example, in terms of data collection, high-density repeated-measures of one or more personality states are needed. Such data can be obtained through ambulatory assessment methods, such as experience sampling methodology (ESM), daily diary research, observational research and research using (physiological) sensors (see Hofmans, De Clercq, Kuppens, Verbeke, \& Widiger, 2019 for an overview of assessing personality dynamics). An important decision in such methods pertains to the number of repeated measurements and the frequency of those measurements. Regarding the number of repeated measurements, studying personality dynamics requires a fairly large number of observations per participant. For example, for testing the PersDyn model through the BHOUM model (see below) one should aim for a minimum of 20 observations per participant on average, and this number increases when more personality dimensions need to be modelled simultaneously. In terms of the frequency of measurement, an important consideration concerns the nature of changes one wishes to observe (i.e., short term fluctuations over a course of the day, or changes that occur over several weeks or months). Practically speaking, current studies on within-person fluctuations in personality have collected reports between five times per day (e.g., Fleeson, 2001) and once every day (e.g., Debusscher, Hofmans, \& De Fruyt, 2016b; Hofmans et al., 2015), while ESM studies on emotions easily involve from 10 to even 50 assessments per day (e.g., Kuppens, Oravecz, \& Tuerlinckx, 2010).

In terms of the statistical analysis, the PersDyn model can be fitted using the Bayesian Hierarchical Ornstein-Uhlenbeck model (BHOUM; Oravecz, Tuerlinckx, \& Vandekerckhove, 2016). The BHOUM model is a model based on stochastic differential equations, expressed by the following equations:

$$
\mathrm{Y}(t)=\Theta(t)+\epsilon(t) \quad \text { (measurement equation) }
$$




$$
d \Theta(t)=\mathrm{B}(\mu-\Theta(t)) d t+\Sigma d \mathrm{~W}(t)
$$

(transition equation)

In the measurement equation, the observed score at time point $t \mathrm{Y}(t)$ is divided into a latent (or true) score $\Theta(t)$, and measurement error $\epsilon(t)$, which is typically normally distributed with an average of zero. In the transition equation, change in the latent score at time point $t d \Theta(t)$ is modeled as a function of (1) the distance between the baseline $\mu$ and the current level $\Theta(t)$, (2) attractor strength B, and (3) a stochastic component $\Sigma d \mathrm{~W}(t)$ that adds noise to the system. In this stochastic component, $\mathrm{W}(t)$ represents the position of a unidimensional Wiener process (or Brownian motion) at time $t$. Such a Wiener process describes a continuous time random (or white noise) process, with this random trajectory being characterized by autocorrelation. As such, the stochastic component $\Sigma d \mathrm{~W}(t)$ can accommodate for the autocorrelation that is often observed in repeated measures data. Focusing on the deterministic part of the transition equation (i.e., $\mathrm{B}(\mu-\Theta(t)) d t$ ), one can see how baseline, variability and attractor strength simultaneously account for within-person fluctuations. If one is currently below his/her personality baseline (i.e., $(\mu-\Theta(t))>0)$, the derivative $(\mu-$ $\Theta(t)) d t$ will be positive, implying that the predicted change at this time point $t$ will be positive. If one is currently above his/her personality baseline (i.e., $(\mu-\Theta(t))<0)$ the derivative $(\mu-\Theta(t)) d t$ will be negative and therefore a decrease in one's state level is predicted. Moreover, when attractor strength B is larger (resp. smaller), one will return to his/her personality baseline faster (resp. slower). A final, yet important characteristic of the BHOUM model is its hierarchical nature, which allows its model parameters-baseline, variability and attractor strength-to vary between individuals.

In terms of model estimation, the BHOUM model makes use of Bayesian estimation (i.e., Markov chain Monte Carlo sampling). Model fit of the BHOUM model can be assessed using a model-comparison strategy in which a series of nested models are compared to each 
other (e.g. a model in which attractor strength is the same across all individuals vs a model in which attractor strength is allowed to vary across individuals). Such competing models can then be compared using the deviance information criterion (DIC). Moreover, also posterior predictive checks are instrumental in checking model fit. The idea behind those posterior predictive checks is that one generates new data based on the fitted model and then compares properties of the generated data with those of the observed data (see Oravecz et al., 2016).

Despite its elegance and direct usefulness for the PersDyn model, an important limitation of the BHOUM model is that it can only be used for modeling one or two personality dimensions. In case higher-dimensional models are needed (for example when one wants to capture complex interactions between more than two personality dimensions), alternative models such as the multidimensional hierarchical bayesian continuous time dynamic model (Driver \& Voelkle, 2018) might be better suited. Although this model has not been applied to personality yet, it certainly represents an important step towards the modeling of complex, multidimensional personality dynamics. Altogether, applying the PersDyn model and higher-dimension models opens up new possibilities in personality research, such as looking at interactions between different traits or facets, comparing temporal changes over various time scales (e.g., daily changes and changes over several months), and testing the predictive validity of the different elements of the model.

\section{Conclusions}

In this paper, we argued that the dynamic approach to personality, as represented by the PersDyn model, has the potential to integrate different perspectives on individual differences. The model offers a straighforward way to capture dynamic changes in personality states through the use of three parameters: trait baseline, trait variability and trait attractor strength. By going beyond the traditional focus on average trait levels, the model has the potential to advance our knowledge on personality processes, including the integration of 
traits and states. Finally, by detailing the factors and processes included in the model, as well as links to existing theories and applications in various research lines, we demonstrated that the dynamic approach to personality offers a consensual paradigm of personality with the potential to advance our understanding and knowledge of individual differences.

\section{References}

Allport, G. W. (1937). Personality: A psychological interpretation. Oxford, England: Holt. Baumeister, R. (1998). The self. In D. Gilbert, S. Fiske, \& G. Lindzey (Eds.), Handbook of social psychology (4th eds., Vol. 2, pp. 680-740). New York: McGraw-Hill.

Bolger, N., \& Schilling, E. A. (1991). Personality and the problems of everyday life: The role of neuroticism in exposure and reactivity to daily stressors. Journal of Personality, 59(3), 355-386. doi: 10.1111/j.1467-6494.1991.tb00253.x

Cervone, D. (2005). Personality architecture: Within-person structures and processes. Annual Review Psycholology, 56, 423-452. doi: 10.1146/annurev.psych.56.091103.070133

Corbetta, D., Vereijken, B. (1999). Understanding development and learning of motor coordination in sport: the contribution of dynamic systems theory. International Journal of Sport Psychology, 30(4), 507-530.

Cramer, A. O. J., Van der Sluis, S., Noordhof, A., Wichers, M., Geschwind, N., Aggen, S. H., Kendler, K. S., \& Borsboom, D. (2012). Dimensions of normal personality as networks in search of equilibrium: you can't like parties if you don't like people. European Journal of Personality, 26, 414-431. doi: 0.1002/per.1866

Dalal, R. S., Meyer, R. D., Bradshaw, R. P., Green, J. P., Kelly, E. D., \& Zhu, M. (2015). Personality strength and situational influences on behavior: A conceptual review and research agenda. Journal of Management, 41(1), 261-287. doi:0.1177/0149206314557524 
Debusscher, J., Hofmans, J., \& De Fruyt, F. (2014). The curvilinear relationship between state neuroticism and momentary task performance. PloS One, 9(9), e106989. doi: 10.1371/journal.pone.0106989

Debusscher, J., Hofmans, J., \& De Fruyt, F. (2016). The effect of state core self-evaluations on task performance, organizational citizenship behaviour, and counterproductive work behaviour. European Journal of Work and Organizational Psychology, 25(2), 301-315. doi: 0.1080/1359432X.2015.1063486

Debusscher, J., Hofmans, J., \& De Fruyt, F. (2016). Do personality states predict momentary task performance? The moderating role of personality variability. Journal of Occupational and Organizational Psychology, 89(2), 330-351. doi:10.1111/joop.12126

Debusscher, J., Hofmans, J., \& De Fruyt, F. (2016). From state neuroticism to momentary task performance: A person $\times$ situation approach. European Journal of Work and Organizational Psychology, 25(1), 89-104. doi:10.1080/1359432X.2014.983085

Debusscher, J., Hofmans, J., \& De Fruyt, F. (2017). The multiple face (t) s of state conscientiousness: Predicting task performance and organizational citizenship behavior. Journal of Research in Personality, 69, 78-85.

doi:10.1016/j.jrp.2016.06.009

DeYoung, C. G. (2015). Cybernetic big five theory. Journal of Research in Personality, 56, 33-58. doi: 10.1016/j.jrp.2014.07.004

Driver, C. C., \& Voelkle, M. C. (2018). Hierarchical Bayesian continuous time dynamic modeling. Psychological Methods. Advance online publication. doi:10.1037/met0000168 
Fleeson, W. (2001). Toward a structure-and process-integrated view of personality: Traits as density distributions of states. Journal of Personality and Social Psychology, 80(6), 1011-1027. doi: 10.1037//0022-3514.80.6.1011

Fleeson, W., \& Gallagher, P. (2009). The implications of Big Five standing for the distribution of trait manifestation in behavior: Fifteen experience-sampling studies and a meta-analysis. Journal of Personality and Social Psychology, 97(6), 1097-1114. doi: $10.1037 / \mathrm{a} 0016786$

Fleeson, W., \& Jayawickreme, E. (2015). Whole trait theory. Journal of Research in Personality, 56(1), 82-92. doi:10.1016/j.jrp.2014.10.009

Furr, R. M. (2009). Personality psychology as a truly behavioural science. European Journal of Personality, 23(5), 369-401. doi: 10.1002/per.724

Hegselman, K., Troitzch, K., \& Muller, U., (Eds.) 1996. Modeling and simulation in the social sciences from the philosophy of science point of view. Dordrecht, The Netherlands: Kluwer Academic.

Hofmans, J., De Clercq, B., Kuppens, P., Verbeke, L., \& Widiger, T. A. (in press). Testing the structure and process of personality using ambulatory assessment data: An overview of within-person and person-specific techniques. Psychological Assessment.

Huang, J. L., \& Ryan, A. M. (2011). Beyond personality traits: A study of personality states and situational contingencies in customer service jobs. Personnel Psychology, 64(2), 451-488. doi: 10.1111/j.1744-6570.2011.01216.x

Huang, J. L., \& Bramble, R. J. (2016). Trait, state, and task-contingent conscientiousness: Influence on learning and transfer. Personality and Individual Differences, 92(5), 180-185. doi: 0.1016/j.paid.2015.12.043 
Jones, A. B., Brown, N. A., Serfass, D. G., \& Sherman, R. A. (2017). Personality and density distributions of behavior, emotions, and situations. Journal of Research in Personality, 69, 225-236. doi: 10.1016/j.jrp.2016.10.006

Johnson, J. G. (2006). Cognitive modeling of decision making in sports. Psychology of Sport and Exercise, 7(6), 631-652. doi: 10.1016/j.psychsport.2006.03.009

Johnson, S. L., \& Nowak, A. (2002). Dynamical patterns in bipolar depression. Personality and Social Psychology Review, 6(4), 380-387. doi: /10.1207/S15327957PSPR0604_12

Judge, T. A., \& Ilies, R. (2002). Relationship of personality to performance motivation: A meta-analytic review. Journal of Applied Psychology, 87(4), 797. doi: 0.1037//00219010.87 .4 .797

Judge, T. A., Simon, L. S., Hurst, C., \& Kelley, K. (2014). What I experienced yesterday is who I am today: Relationship of work motivations and behaviors to within-individual variation in the five-factor model of personality. Journal of Applied Psychology, 99(2), 199-221. doi: 10.1037/a0034485

Kelso, J.A.S. (1995). Dynamic patterns: The self-organization of brain and behavior. Cambridge, MA: MIT Press

Kuppens, P., Oravecz, Z., \& Tuerlinckx, F. (2010). Feelings change: Accounting for individual differences in the temporal dynamics of affect. Journal of Personality and Social Psychology, 99(6), 1042-1060. doi: 10.1037/a0020962

Lee, K., \& Ashton, M. C. (2004). Psychometric properties of the HEXACO personality inventory. Multivariate Behavioral Research, 39(2), 329-358.

Doi:10.1207/s15327906mbr3902_8 
Lewis, M. D. (2005). Bridging emotion theory and neurobiology through dynamic systems modeling. Behavioral and Brain Sciences, 28(2), 169-194. doi:10.1017/S0140525X0500004X

Magnus, K., Diener, E., Fujita, F., \& Pavot, W. (1993). Extraversion and neuroticism as predictors of objective life events: A longitudinal analysis. Journal of Personality and Social Psychology, 65(5), 1046. doi: 10.1037/0022-3514.65.5.1046

Mischel, W., \& Shoda, Y. (1995). A cognitive-affective system theory of personality: reconceptualizing situations, dispositions, dynamics, and invariance in personality structure. Psychological Review, 102(2), 246-269. doi: 10.1037/h0037130

Mischel, W., \& Shoda, Y. (1998). Reconciling processing dynamics and personality dispositions. Annual Review of Psychology, 49(1), 229-258. doi:10.1146/annurev.psych.49.1.229

Mischel, W., \& Shoda, Y. (1999). Integrating dispositions and processing dynamics within a unified theory of personality: The cognitive-affective personality system. In L. A. Pervin \& O. P. John (Eds.), Handbook of Personality: Theory and Research (pp. 197218). New York, NY, US: Guilford Press.

McCrae, R. R., \& Costa, P. T., Jr. (1999). A Five-Factor theory of personality. In L. A. Pervin \& O. P. John (Eds.), Handbook of Personality: Theory and Research (pp. 139153). New York, NY, US: Guilford Press.

Mõttus, R., Epskamp, S., \& Francis, A. (2017). Within-and between individual variability of personality characteristics and physical exercise. Journal of Research in Personality, 69, 139-148. Doi: 10.1016/j.jrp.2016.06.017

Nowak, A., Urbaniak, J., \& Zienkowski, L. (1994). Clustering processes in economic transition. Research Bulletin, 3(3), 43-61. 
Nowak, A., Vallacher, R. R., Tesser, A., \& Borkowski, W. (2000). Society of self: The emergence of collective properties in self-structure. Psychological Review, 107, 3961. doi: $10.1037 / \mathrm{h} 0046049$

Nowak, A., Vallacher, R. R., \& Zochowski, M. (2005). The emergence of personality: Dynamic foundations of individual variation. Developmental Review, 25(3-4), 351385. doi:10.1016/j.dr.2005.10.004

O'Connor, E. (2010). Teacher-child relationships as dynamic systems. Journal of School Psychology, 48(3), 187-218. doi: 10.1016/j.jsp.2010.01.001

Oravecz, Z., Tuerlinckx, F., \& Vandekerckhove, J. (2016). Bayesian data analysis with the bivariate hierarchical Ornstein-Uhlenbeck process model. Multivariate Behavioral Research, 51(1), 106-119. doi: 10.1080/00273171.2015.1110512

Orom, H., \& Cervone, D. (2009). Personality dynamics, meaning, and idiosyncrasy: Identifying cross-situational coherence by assessing personality architecture. Journal of Research in Personality, 43(2), 228-240. Doi: 10.1016/j.jrp.2009.01.015

Read, S. J., Monroe, B. M., Brownstein, A. L., Yang, Y., Chopra, G., \& Miller, L. C. (2010). A neural network model of the structure and dynamics of human personality. Psychological Review, 117(1), 61-92. doi: 10.1037/a0018131

Revelle, W., \& Condon, D. M. (2015). A model for personality at three levels. Journal of Research in Personality, 56, 70-81. doi: 10.1016/j.jrp.2014.12.006

Sadler, P., Woody, E., McDonald, K., Lizdek, I., \& Little, J. (2015). A lot can happen in a few minutes: Examining dynamic patterns within an interaction to illuminate the interpersonal nature of personality disorders. Journal of Personality Disorders, 29(4), 526-546. doi: 10.1521/pedi.2015.29.4.526

Saucier, G. (1994). Mini-Markers: A brief version of Goldberg's unipolar Big-Five markers. Journal of Personality Assessment, 63(3), 506-516.doi:10.1037/t07015-000 
Shoda, Y., LeeTiernan, S., \& Mischel, W. (2002). Personality as a dynamical system: Emergence of stability and distinctiveness from intra and interpersonal interactions. Personality and Social Psychology Review, 6(4), 316-325. doi: 10.1207/S15327957PSPR0604_06

Sosnowska, J. \& Hofmans, J., \& De Fruyt, F. (2016, July). Relating neuroticism to emotional exhaustion: a dynamic approach to personality. Paper presented at the 18th European Conference on Personality, Timisoara, Romania.

Sosnowska, J. \& Hofmans, J. (2018, March). Revisiting the neuroticism-performance link: a dynamic systems approach. Paper presented at the conference of the Society for Personality and Social Psychology, Warsaw, Poland.

Sosnowska, J., Hofmans, J., \& De Fruyt, F. (2019). Relating emotional arousal to work vigour: A dynamic systems perspective. Personality and Individual Differences. 136(1), 178-183. doi: 10.1016/j.paid.2017.06.040

Sosnowska, J., Hofmans, J., \& Lievens, F. (in press). Assessing personality dynamics in personnel selection. In J. F. Rauthmann (Ed.). The Handbook of Personality Dynamics and Processes. Elsevier.

Steenbeek, H., \& van Geert, P. (2013). The emergence of learning-teaching trajectories in education: A complex dynamic systems approach. Nonlinear dynamics, psychology, and life sciences, 17(2), 233-267.

Thelen, E., Skala, K., \& Kelso, J.A.S. (1987). The dynamic nature of early coordination: Evidence from bilateral leg movements in young infants. Developmental Psychology, 23(2), 179-186. doi: 10.1037/0012-1649.23.2.179

Vallacher, R. R., \& Nowak, A. (2007). Dynamical social psychology: Finding order in the flow of human experience. In A. W. Kruglanski \& E. T. Higgins (Eds.), Social 
Psychology: Handbook of Basic Principles (Vol. 2, pp. 734-758). New York: Guilford Publications

Vallacher, R. R., Nowak, A., Froehlich, M., \& Rockloff, M. (2002). The dynamics of selfevaluation. Personality and Social Psychology Review, 6(4), 370-379. doi: 10.1207/S15327957PSPR0604_11

Van Vugt, M., \& Hart, C. M. (2004). Social identity as social glue: the origins of group loyalty. Journal of Personality and Social Psychology, 86(4), 585-598. doi: $10.1037 / 0022-3514.86 .4 .585$

Wright, A. G., Hopwood, C. J., \& Simms, L. J. (2015). Daily interpersonal and affective dynamics in personality disorder. Journal of Personality Disorders, 29(4), 503-525. doi: 10.1521/pedi.2015.29.4.503

Table 1. Comparison of the traditional vs. dynamic approach to personality.

\section{The traditional approach to personality The dynamic systems approach to} personality

\section{General assumptions}

Personality is a set of predispositions

Personality is a sum of traits (synthesis)

Focus on individual components of

personality (traits and their facets)

Focus on predetermination and causality deterministic approach
Personality is a dynamic system

Personality is more than the sum of traits due to dynamic interactions between the elements (analysis)

Focus on interactions between the components of personality Focus on growth and novelty - probabilistic approach 
personality

\section{Structure}

Focus on between-person differences,

Focus on both within- and between-person

within-person differences are either ignored differences

or treated as measurement error

Different levels of personality are redundant Each level of personality brings a distinctive

contribution

\section{Person $\mathrm{x}$ situation $\mathrm{x}$ behaviour triad}

Top-down approach: traits predict

behaviours

Traits are invariant across situations

Linear relationships
Bottom-up approach: traits emerge from

states and behaviours

A feedback loop between person and situations

Non-linear relationships and changes over

time

\section{The role of time}

The temporal dimension is ignored
The temporal dimension is essential to

understanding personality

Change only over long periods of time (i.e.,

maturation)

Omits the role of self-regulation

Each behavioural manifestation is isolated
Change over time (short- and long-term change)

Crucial role for self-regulation

Each behavioural manifestation depends on the previous one (path dependency) 
Figure 1. Hypothethical trajectories in PersDyn model.

1.1. high baseline, high variability, high attractor strength $\quad 1.2$ high baseline, high variability, low attractor strength
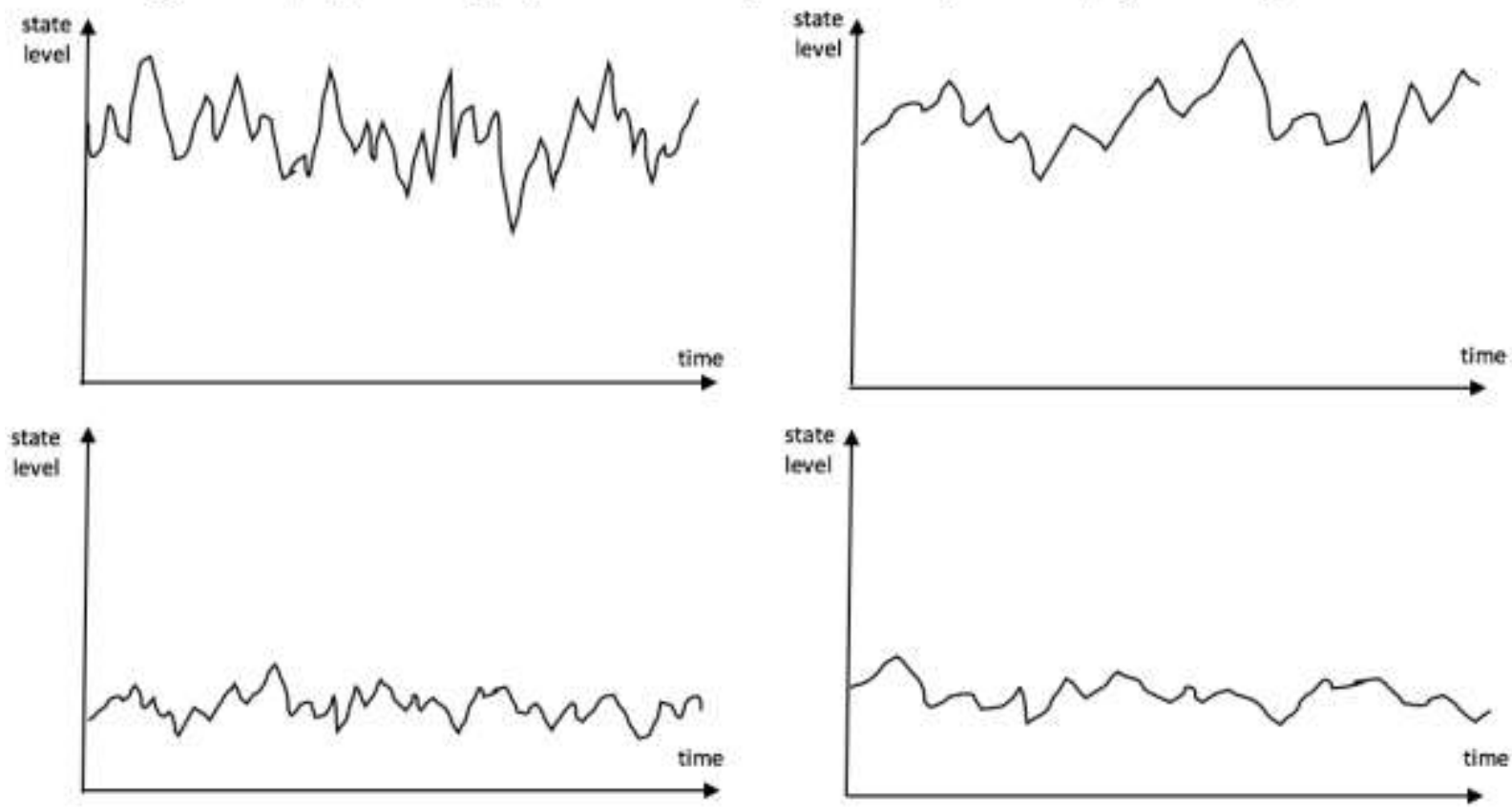

1.3. low baseline, low variability, high attractor strength

1.4. low baseline, low variability, low attractor strength 
Figure 2. Bottom-up vs. top-down approach in personality.
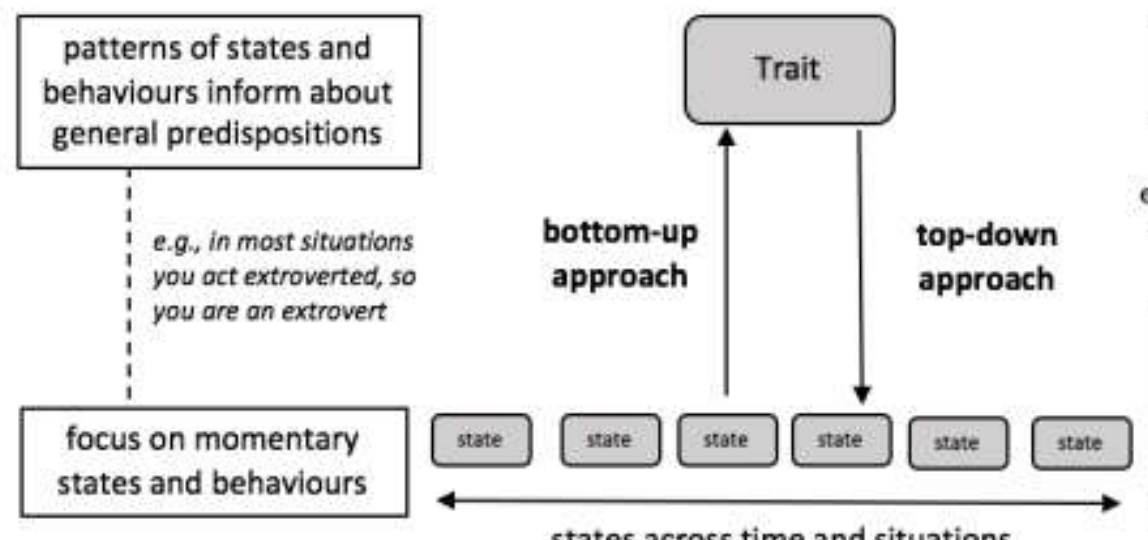

focus on traits and general predispositions

e.g. you are an extrovert, so you act extroverted in most situations

general predispositions inform about momentary states and behaviours 